\title{
Simulation of the Processing Process in a Glow Discharge Plasma Generator
}

\author{
Vladimir A. Logvin ${ }^{1, *}$, Ludmila A. Yvarova ${ }^{2}$ \\ ${ }^{1}$ Interstate educational institution of the higher education "Belarusian-Russian University", BL-212000, Mogilev, Republic of Belarus \\ doctoral candidate Institute of design-technology informatics Russian Academy of Sciences Moscow, Russia \\ ${ }^{2}$ Moscow State Technological University "STANKIN", RU-127055, Moscow, Russia
}

\begin{abstract}
A theory has been developed that explains the structure formation in a thin surface layer of a material after low-energy exposure to a glow discharge plasma. This makes it possible to design new methods, technologies and automated devices for the development of an automated technological environment for strengthening various types of tools from various materials. Based on the developed theory, a self-learning system with a developing database was created for monitoring all stages of processing in a glow discharge plasma generator. Any crystal structure can be classified as a complex nonlinear system. When studying the dynamic response of such systems to an external low-energy effect, it was shown that by the time when the nonlinear vibrations stop, the atoms of the crystal lattice are stabilized in new positions. Based on the purpose of the products to be processed in the glow discharge plasma generator, with taking into account their operating conditions, it is possible to increase their production life up to 2 times or more. These technologies should be used at the final stage of the production process because they do not lead to distortion of the shape and size of the working surfaces of products being manufactured. In this case, the residual stresses are redistributed in the surface layer of the material with the formation of an equal structure that improves the running-in conditions of mating parts of devices and mechanisms. For cutting and forming tools, the run-in stage is reduced to the stage of uniform wear.
\end{abstract}

Keywords: plasma, glow discharge, control system, automated technological environment, modeling.

\section{Introduction}

An urgent problem of mechanical engineering is to ensure stable growth in the quality of manufactured products and, thus, to increase their competitiveness. The development of new technologies that ensure the formation of the necessary physical and mechanical properties of the surface layer of tool materials, adapted for use in an automated technological environment, has become an increasingly urgent problem of modern tool production.

Energy-efficient methods of ion-plasma processing that improve the functional properties of tools and other crystal structures include the action of glow discharge plasma formed in controlled technological environments $[1,2]$. Structural changes appear in the surface layer of materials under the influence of a wide range of energy flows. The energy flux of glow discharge plasma radiation particles that initiate structural transformations causes changes in the physical and mechanical properties of the surface material of the products being strengthened. Structural transformations occur in the surface layer of materials under the influence of a wide range of glow discharge plasma radiation energies, which cause changes in their properties.

The formation of the desirable functional properties is ensured by using self-learning systems with a developing database for monitoring all stages of product processing in the glow discharge plasma generator. Based on the analysis and evaluation of the quality of functioning of the real object to be processed, it is possible to ensure the stability of the quality of operational management and, thus, the formation of the specified properties of products being processed. The energy of the particles formed in the discharge must correspond to the energy that ensures the active formation of point defects in the material surface layers. Such technologies are based on nonlinear effects inherent in the potential energy of interaction between structural elements of crystal lattices. Based on this, their structuring is provided under the low-energy effect of glow discharge plasma radiation. It is important to establish the nonlinear characteristics of the processed materials. This makes it possible to provoke internal rearrangements of systems with the formation of new metastable stable structures with minimal energy impact. These structures can be spatial, temporal, or spatiotemporal. The tendency of open systems to form autonomous time and space-time structures as a result of external energy influence is identified as selforganization of systems [3-8].

The treatment of ready-to-use products with glow discharge plasma radiation makes it possible to optimize the advantages of the processes of interaction of incoming charged particles with the surface of various materials. This allows achieving the required properties

e-mail: logvinvladim@yandex.ru 
by varying particle energy, radiation dose, temperature of irradiated objects, materials of electrodes and medium formed in the interelectrode gap [1, 2]. The radiation of the plasma glow discharge has a wide range of ion flux in ions and their energy. This does not require special formation and concentration of ion fluxes. This process can be classified as irradiation by a chaotic flow of incoming ions of the crystal lattice of materials. Since the development of technologies based on active, longrange nanostructuring by glow discharge plasma irradiation depends on the processes occurring in the interelectrode gap, the physical nature of these processes should be taken into account. Thus, it is important to take into account the physics behind these processes, especially for those occurring in the cathode dark space.

The main objective of this work is to develop a theory that can simulate the functioning of a control system for fast-flowing processes in a glow discharge plasma generator. This will allows designing new methods, technologies and automated devices that can be used in automated technological environments.

\section{Method of research}

Controlled formation and burning of glow discharge in the plasma generator in controlled technological environments, used for processing various materials for the purpose of changing in the surface layer physical and mechanical properties is an important condition for efficient reproducibility of processing results. To simulate these processes let's use the position of quantum mechanics which consists in the, that any system can be described by setting in the general case a complex wave function of the form. Ability to detect a charged particle at the moment time $t$ at some point near-cathode space closed volume the plasma generator with the radius vector is determined by the probability density, which is represented by the square of the modulus of the wave function. Probability of finding a particle in an elementary volume working near-cathode space the plasma generator at a point with a radius vector at time $\mathrm{t}$ can be represented as

$$
P(\overrightarrow{\mathbf{r}}, t) d^{2} \overrightarrow{\mathbf{r}}=C^{2}|\psi(\overrightarrow{\mathbf{r}}, t)|^{2} d^{3} \overrightarrow{\mathbf{r}},
$$

where $\mathrm{C}$ - is the constant accepted from the condition

$$
\int_{-\infty}^{+\infty} \psi^{*}(\overrightarrow{\mathbf{r}}, t) \psi(\overrightarrow{\mathbf{r}}, t) d^{3} \overrightarrow{\mathbf{r}}=1,
$$

where $\psi^{*}(\overrightarrow{\mathbf{r}}, t)$ - function complex-conjugate $\psi(\overrightarrow{\mathbf{r}}, t)$.

Changes in function time $\psi(\overrightarrow{\mathbf{r}}, t)$ on condition, that the particle is moving in potential $U(\overrightarrow{\mathbf{r}}, t)$ they are reflected by the non-stationary Schrodinger equation [9] of the following form

$$
i \square \frac{\partial \psi(\overrightarrow{\mathrm{r}}, t)}{\partial t}=-\frac{\square^{2}}{2 m} \Delta \psi(\overrightarrow{\mathrm{r}}, t)+U(\overrightarrow{\mathrm{r}}, t) \psi(\overrightarrow{\mathrm{r}}, t),
$$

where $m$ - is the mass of the particle, $\square-$ Planck constant divided by $2 \pi$

$$
\Delta=\frac{\partial^{2}}{\partial x^{2}}+\frac{\partial^{2}}{\partial y^{2}}+\frac{\partial^{2}}{\partial z^{2}}-\text { operator Laplace. }
$$

In the one-dimensional case, the Schrödinger equation is represented in the following form

$$
i \square \frac{\partial \psi(x, t)}{\partial t}=-\frac{\square^{2}}{2 m} \frac{\partial^{2}}{\partial x^{2}} \psi(x, t)+U(x, t) \psi(x, t) .(4)
$$

Provided that the potential does not depend on time, the solution of equation (4) can be performed in the following form

$$
\psi(x, t)=\varphi(x, t) e^{-\frac{i E t}{\square}} .
$$

If we assume that the particle arrives in the state described by the wave function (5), it must have a certain amount of energy E. When substituting (5) in (4) and performing transformations, we have a stationary Schrodinger equation of the form

$$
\left[-\frac{\square^{2}}{2 m} \frac{\partial^{2}}{\partial x^{2}}+U(x)\right] \varphi(x)=E \varphi(x),
$$

where $-\frac{\hbar^{2}}{2 m} \frac{\partial^{2}}{\partial x^{2}}+U(x)$ energy operator $\widehat{H}$ or the Hamilton operator [7-10],

$\varphi(x)$ - eigenfunction of the Hamiltonian,

$E_{n}$ - operator's own value $\widehat{H}$.

As a result, equation (6) can be written as

$$
\widehat{H} \varphi(x)=E \varphi(x) .
$$

Due to the fact that the operator $\widehat{H}$ it can have $\mathrm{n}$ proper functions $\varphi_{n}$ and $\mathrm{n}$ corresponding energy values $E_{n}$, if they coincide, we get degenerate States, and if there are many continuous values, a continuous spectrum is formed. The stable position of any particle is considered to be the state of equilibrium or when the particle has the lowest energy reserve $E_{1}$ [9-14].

When the potential is stable $U(x)=$ const

$$
\begin{gathered}
{\left[-\frac{\square^{2}}{2 m} \frac{\partial^{2}}{\partial x^{2}}+U(x)\right] \phi(x)-E \phi(x)=0} \\
\frac{\partial^{2} \phi(x)}{\partial x^{2}}-\frac{2 m}{\square^{2}}(U(x)-E) \phi(x)=0
\end{gathered}
$$
get:

After performing the appropriate transformations, we

$$
\lambda^{2}-\frac{2 m}{\square^{2}}(\boldsymbol{U}(\boldsymbol{x})-\boldsymbol{E})=\mathbf{0} .
$$

When solving the presented equation, two directions are possible:

When

$$
U(x)<E, U(x)-E<0
$$

and

$$
\lambda_{1,2}=\frac{\sqrt{2 m}}{\square} i \sqrt{E-U(x)},
$$

and when $U(x)>E, U(x)-E>0$ in this case, the necessary effect may not be because the impact of a charged particle on the material of the working surface of the tool being strengthened will be minimal or close to zero. As a 
result, we will analyze the first direction in solving the problem and:

$$
\begin{aligned}
& \phi(x)= \\
& C_{1} \cos \frac{\sqrt{2 m}}{\hbar} \sqrt{E-U(x)} x++C_{2} \sin \frac{\sqrt{2 m}}{\hbar} \sqrt{E-U(x)} x, \\
& \text { at } \mathbf{x}_{\min }=\mathbf{0} \phi\left(\mathbf{x}_{\min }\right)=\left(\mathrm{C}_{1} \cos 0+\mathrm{C}_{2} \sin 0\right)= \\
& \mathbf{0}, \mathrm{C}_{\mathbf{1}}=\mathbf{0} \text {, } \\
& \text { at } \mathbf{x}_{\text {max }}=0 \\
& \phi\left(x_{\text {max }}\right)=\left(C_{2} \sin \frac{\sqrt{2 m}}{\hbar} \sqrt{E-U(x)} x_{\text {max }}\right. \\
& =\mathbf{0}, \frac{\sqrt{2 \mathbf{m}}}{\hbar} \sqrt{\mathbf{E}-\mathbf{U}(\mathbf{x})} \mathbf{x}_{\max }=\pi \mathbf{n}, \mathbf{E}_{\mathbf{n}} \\
& \left.=\mathbf{U}(\mathbf{x})+\frac{\pi^{2} \mathbf{n}^{2} \hbar^{2}}{2 \mathbf{m} \mathbf{x}_{\max }^{2}}\right) \\
& \phi_{\mathbf{n}}(\mathrm{x})=\mathrm{C}_{2} \sin \frac{\pi \mathrm{nx}}{\mathrm{x}_{\max }} \\
& \Psi=\sum_{n=1}^{\infty} B_{n} e^{\frac{-i\left(U(x)+\frac{\pi^{2} n^{2} \hbar^{2}}{2 m x_{\max }^{2}}\right) t}{\hbar}} \sin \frac{\pi n x}{x_{\max }},
\end{aligned}
$$

where $B_{n}=\frac{2}{x_{\max }} \int_{0}^{x_{\max }} \Psi(0) \sin \frac{\pi n x}{x_{\max }} d x$ - the

Fourier coefficients.

$$
\Psi(0)=\Psi(t=0) .
$$

Taking into account the fact that the mass of incident ions involved in interaction with the processed tools in the glow discharge plasma generator is different and the mass distribution is also different, we will use the exponential distribution. Then the total mass of the particles involved in the interaction with the treated surface will be

$$
\Psi_{\Sigma} \int_{m_{\min }}^{m_{\max }} \boldsymbol{\psi}(\boldsymbol{m}) \boldsymbol{f}(\boldsymbol{m}) d \boldsymbol{m} .
$$

After performing substitutions and transformations, we get

$$
\begin{gathered}
\Phi(\mathbf{x})=\frac{\sqrt{2 \mathbf{m}}}{\hbar} \sqrt{\mathbf{E}-\mathbf{b}-\mathbf{a x}}\left(\mathbf { C } _ { 2 } \mathrm { J } _ { \frac { 1 } { 3 } } \left(-\frac{\sqrt{2 \mathrm{~m}}}{\hbar \mathbf{a}}(\mathrm{E}-\mathbf{b}\right.\right. \\
\left.--\mathbf{a x})^{\frac{3}{2}}\right)+\mathrm{C}_{1} \mathrm{~J}_{\frac{-1}{3}}\left(-\frac{\sqrt{2 \mathrm{~m}}}{\hbar \mathbf{a}}(\mathbf{E}-\mathbf{b}\right. \\
\left.-\mathbf{a x})^{\frac{3}{2}}\right)
\end{gathered}
$$

where $J_{\frac{1}{3}}$ even and $J_{\frac{-1}{3}}$ odd - Bessel functions.

Thus

$$
\begin{gathered}
\Phi(x)=\frac{\sqrt{2 m}}{\hbar} \sqrt{E-b-a x}\left(C _ { 2 } J _ { \frac { 1 } { 3 } } \left(\frac{\sqrt{2 m}}{\hbar a}(E-b-\right.\right. \\
\left.a x)^{\frac{3}{2}}\right)+C_{1} J_{\frac{-1}{3}}\left(\frac{\sqrt{2 m}}{\hbar a}(E-b-a x)^{\frac{3}{2}}\right)
\end{gathered}
$$

Taking into account the boundary conditions, we perform the substitution and get

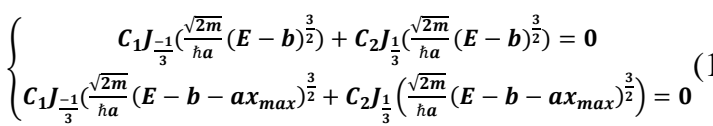

If the solution is trivial, the main determinant of the system must be zero

$$
\begin{array}{r}
\Delta=\mathbf{J}_{\frac{-1}{3}}\left(\frac{\sqrt{2 \mathbf{m}}}{\hbar \mathbf{a}}(\mathbf{E}-\mathbf{b})^{\frac{3}{2}}\right) \mathbf{J}_{\frac{1}{3}}\left(\frac{\sqrt{2 \mathbf{m}}}{\hbar \mathbf{a}}\left(\mathbf{E}-\mathbf{b}-\mathbf{a} \mathbf{x}_{\max }\right)^{\frac{3}{2}}\right)- \\
-\mathbf{J}_{\frac{-1}{3}}\left(\frac{\sqrt{2 \mathbf{m}}}{\hbar \mathbf{a}}\left(\mathbf{E}-\mathbf{b}-\mathbf{a x} \mathbf{x}_{\max }\right)^{\frac{3}{2}}\right) \mathbf{J}_{\frac{1}{3}}\left(\frac{\sqrt{2 \mathbf{m}}}{\hbar \mathbf{a}}(\mathbf{E}-\mathbf{b})^{\frac{3}{2}}\right)=\mathbf{0} .
\end{array}
$$

Equation (11) is a condition for determining the $E_{n}$ and its solution can be represented as

$$
\begin{aligned}
\Psi \sum_{n=1}^{\infty} e^{\frac{-i E_{n} t}{\hbar}}\left(B_{1 n} J_{\frac{-1}{3}}\left(\frac{\sqrt{2 m}}{\hbar a}\left(E_{n}-b-a x\right)^{\frac{3}{2}}\right)\right. \\
++B_{2 n} J_{\frac{1}{3}}\left(\frac{\sqrt{2 m}}{\hbar a}\left(E_{n}-b-a x\right)^{\frac{3}{2}}\right)
\end{aligned}
$$

Then

$$
\mathbf{E}_{\mathbf{n}}=\left(\frac{\boldsymbol{\mu}_{\mathbf{n}} \hbar \mathbf{a}}{\sqrt{2 \mathbf{m}}}\right)^{\frac{2}{3}}+\mathbf{b}
$$

For each $E_{n}$ set the possible sets $x_{\max }$

$$
\mathbf{x}_{\max \mathbf{j}}=\mathbf{E}_{\mathbf{n}}-\mathbf{b}-\left(\boldsymbol{\mu}_{\mathbf{j}}\left(\mathbf{E}_{\mathbf{n}}\right) \frac{\hbar \mathbf{a}}{\sqrt{\mathbf{2 m}}}\right)^{\frac{2}{3}} .
$$

This allows you to match xmaxj and their corresponding possible values En. Due to the fact that the arguments of the Bessel function are much larger than 1, it is more convenient to operate with dimensionless quantities, and for this we will use asymptotic formulas, which for our case will take the form

$$
\begin{aligned}
& J_{\frac{1}{3}}(x) \approx \sqrt{\frac{2}{\pi x}} \cos \left(x-\frac{5 \pi}{12}\right) \\
& J_{\frac{-1}{3}}(x) \approx \sqrt{\frac{2}{\pi x}} \cos \left(x-\frac{\pi}{12}\right)
\end{aligned}
$$

From equation (11), after substitutions, we obtain

$$
\frac{\cos \left(\frac{\sqrt{2 m}}{\hbar a}(E-b)^{\frac{3}{2}}-\frac{\pi}{12}\right)}{\cos \left(\frac{\sqrt{2 m}}{\hbar a}(E-b)^{\frac{3}{2}}-\frac{5 \pi}{12}\right)}=\frac{\cos \left(\frac{\sqrt{2 m}}{\hbar a}\left(E-b-a x_{\max }\right)^{\frac{3}{2}}-\frac{\pi}{12}\right)}{\cos \left(\frac{\sqrt{2 m}}{\hbar a}\left(E-b-a x_{\max }\right)^{\frac{3}{2}}-\frac{5 \pi}{12 \hbar a}\right)}
$$

As a result of solving equation (12), we obtain the values $E_{n}$.

In the general case, we denote

$$
\begin{gathered}
Z=\frac{\sqrt{2 m}}{\hbar a}\left(E_{n}-b\right)^{\frac{3}{2}} \\
\alpha=\frac{\left(E-b-a x_{\max }\right)^{\frac{3}{2}}}{(E-b)^{\frac{3}{2}}}
\end{gathered}
$$


Making substitutions from (10), we obtain

$$
\frac{\frac{J_{-1}}{3}(Z)}{J_{\frac{1}{3}}(Z)}=\frac{\frac{J_{-1}}{3}(\alpha Z)}{J_{\frac{1}{3}}(\alpha Z)}
$$

Solving equation (13) we find the first few roots for different $\alpha$ and $Z$. Then we can determine different values $E_{n}$ for $\Pi=0,1,2 \ldots$. obtain

From equation (12), performing transformations, we

$$
\frac{\sqrt{2 m}}{\hbar a}(E-b)^{\frac{3}{2}}=\frac{\pi n(E-b)^{\frac{3}{2}}}{(E-b)^{\frac{3}{2}}-\left(E-b-a x_{\max }\right)^{\frac{3}{2}}}
$$

As a result of the transformations carried out, a dependence was obtained for determining the energy of ions formed in the plasma generator of a glow discharge in accordance with their mass andE $\mathrm{E}_{\mathrm{n}}$

$$
(E-b)^{\frac{3}{2}}-\left(E-b-a x_{\max }\right)^{\frac{3}{2}}=\frac{\hbar a \pi n}{\sqrt{2 m}}
$$

\section{Results and discussion}

Controlled formation of the ion flux in the glow discharge plasma generator was made possible due to the obtained analytical dependences for determining the energy of charged particles in accordance with their mass. The formation of charged particles in the interelectrode gap with a certain mass and energy is random. Using the Schrodinger equation to solve this problem is most appropriate. As a result of modeling, it is established that depending on the technological medium used and the rate of its pumping, the necessary ion flow can be formed. This flow has a wide range of masses and energies, which is able to perform appropriate transformations in the surface layer of the processed products. The use of self-learning systems with a developing database for monitoring all stages of product processing in a glow discharge plasma generator ensures its controlled formation and Gorenje. In controlled technological environments in a plasma generator, glow discharge combustion is characterized by the highest stability of reproducible results.

As a result of electron microscopic study of the fine structure of materials exposed to glow discharge plasma radiation, it was found that low-energy exposure to metal samples leads to an increase in the dislocation density at a depth exceeding the projective range of ions. This effect is called long-range $[15,16]$.

A significant increase in the dislocation density is observed in well-annealed samples with a low initial dislocation density $(\alpha-\mathrm{Fe})$. In fig. 1 , a shows the dislocation structure of $\alpha$-Fe before irradiation, and Fig. $1 \mathrm{~b}$ after low-energy exposure to glow discharge plasma. It was found that the effect of a glow discharge plasma causes an increase in the dislocation density to a depth of $8.19 \mathrm{~mm}$ from the irradiation surface, and the structure corresponds to a deeply deformed state of the material [17].

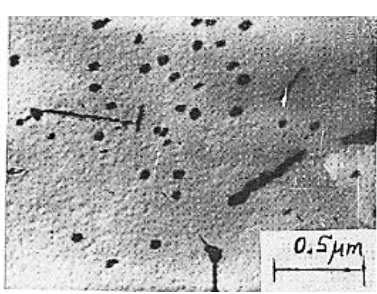

a)

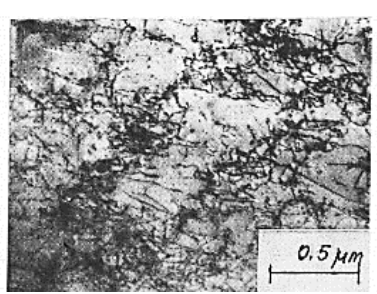

b)
Figure: 1. Dislocation structure of $\alpha$-Fe: a - unirradiated state; $\mathrm{b}$ - after irradiation in a glow discharge at a voltage of $1 \mathrm{kV}$ at a distance of $8.19 \mathrm{~mm}$ from the irradiation surface

Crystalline atomic structures can be classified as complex nonlinear systems. In the study of the dynamic response of such systems to an external low-energy impact, it was shown that by the time the nonlinear oscillations stop, the atoms of the crystal lattice are stabilized in new positions. The time period required for this stabilization is several orders of magnitude higher than the time of ordinary atomic relaxation. In this case, long-lived metastable structural states of the crystal lattice are formed. The type of the formed heterogeneous structures depends on the potential describing interatomic bonds and the magnitude of the external influence. The nonlinearity coefficients and additional states in multiwell potentials characterizing interatomic bonds depend on the type of potential or the properties of the irradiated material. Thus, for the Morse potential with the nonlinearity parameters established for $\alpha$-Fe, it was shown that an external low-energy action causes the formation of solitons in nonlinear lattices moving along certain atomic chains [16]. Undamped pulsations during the motion of a soliton are transmitted to a depth exceeding the value of the projective path from the irradiated surface. In real crystal structures, dislocations are analogous to solitons. An increase in the dislocation density at sufficiently large distances from the irradiation surface is experimentally confirmed within the framework of the nonlinear theory. The displacement of atoms and their stabilization in the final state is the result of nonlinear collective vibrations of all atoms of the lattice after a low-energy impact. Therefore, by means of a weak external influence, it is possible to initiate internal processes in systems with the formation of new long-lived structural states. Thus, as a result, the crystal lattice represents an active zone for a long time.

\section{Conclusion}

The quality of the performed simulations was assessed when manufacturing products in a glow discharge plasma generator. It is shown that depending on the technological environment used two effects, short-range and long-range, were observed after the exposure to plasma. The short-range effect is caused by ion current transfer and leads to changes in the chemical and phase composition of the surface layer, modification and refinement of the crystal structure, and amorphization of the thin surface layer.

The long-range effect is manifested in the fact that the process of generating defects during processing in a 
glow discharge shows the features of a dissipative process with elements of self-organization and has a quasi-oscillating nature. Obviously, there is a variable amplifying effect of either the surface layers of the material or its volume. Defect density waves propagate through the volume of the material, which characterizes the process as a synergistic one. In this case, there is an oscillatory change in the dislocation density over time. Early fragmentation confirms the presence of oscillations in the dislocation subsystem and a multiparametric oscillatory process occurs.

The treatment of metals and alloys with low-energy glow discharge plasma leads to an increase in the dislocation density to a depth significantly exceeding the projective range of ions from the target surface. This phenomenon can be classified as a long-range effect.

\section{Acknowledgments}

This work was partially supported by the RSF project No. 18-11-00247. This work was carried out using equipment provided by the Center of Collective Use of MSUT "STANKIN".

\section{References}

1. I.V. Tereshko, V.A. Logvin, V.M. Tereshko, S.A. SHeptunov, Bulletin of the Bryansk State Technical University, 3(51) (2016) DOI: $10.12737 / 22066$

2. I.V. Tereshko, V.A. Logvin, V.M. Tereshko, S.A. SHeptunov, V.P. Red'ko, Proc. 6th Int. Konf. «Design and technological informatics», 21-29 (2017)

3. G. Haken, Secrets of nature. Synergetics: the study of interaction (Moscow-Izhevsk. Institute for Computer Research, 2003)

4. V.K. Vanag, Dissipative structures in reactiondiffusion systems (Experiment and theory, 2008)

5. Yu.P. Haraev, Polzunovsky almanac, 4, 47-51 (2004)

6. W. Möller, Fundamentals of Ion-Surface Interaction (Technical University of Dresden, 2004)

7. I.A. Hamad, D. Robb, P.A. Rikvold, Computer Simulation Studies in Condensed-Matter Physics XIX. Proceedings of the Nineteenth Workshop, 8993 (2006) https://doi.org/10.1007/978-3-540-85625-2_14

8. W. Eckstein, Computer Simulation of Ion-Solids Interaction (Springer-Verlag, Berlin, Heidelberg, New York, 1991)

9. L.D. Landau, E.M. Lifshic, Quantum mechanics (Non-relativistic theory. Moscow. 2004)

10. V.F. Zajcev, A.D. Polyanin, Directory. Ordinary differential equations (Moscow., 2001).
11. G. Korn, The Mathematics Handbook for Scientists and Engineers: Definitions, Theorems, Formulas (2014)

12. N.M. Chernavskaya, D.S. Chernavskii, L.A. Uvarova, Mathematical Models of Non-Linear Excitations, Transfer, dynamics, and Control in Condensed Systems and Other Media, 181-194, (1999) https://doi.org/10.1007/978-1-4615-4799-0_16

13. B.N. Aleksić, L.A. Uvarova, Opt Quant Electron, 52, 175 (2020). https://doi.org/10.1007/s11082-020-02271-2

14. L.A. Uvarova, Y. Burenok, International Journal of Pure and Applied Mathematics, 109(3), 691-707 (2016) doi:10.12732/ijpam.v109i3.17

15. I.V. Tereshko, Proc. 4 th all Russian Conf. with international participation of the scientific and technical confirence 2005, 210-215 (2005)

16. I.V. Tereshko, V.A. Logvin, V. Red'ko, Bulletin of the Belarusian-Russian University, 1, 59-70 (2013) [in Russian]

17. I.V. Tereshko,. In: Application of Particle and Laser Beams in Materials Technology, 595-602 (1995) https://doi.org/10.1007/978-94-015-8459-3_41 